\title{
Intrinsic top quark properties - top mass, charge and polarization
}

\author{
Matteo Franchini ${ }^{1}$ On behalf of the ATLAS Collaboration \\ University of Bologna \& INFN \\ Via Zamboni, 33 - 40126 Bologna, ITALY \\ matteo.franchini@cern.ch
}

Properties of the top quark are measured with the ATLAS detector using LHC proton-proton collisions data. Measurements of the top-quark mass and charge, as well as of the polarization of $\mathrm{W}$ bosons in top quark decays to probe the Wtb-vertex are presented.

XXI International Workshop on Deep-Inelastic Scattering and Related Subjects April 22-26, 2013

Marseilles, France

1

Matteo Franchini 


\section{Introduction}

High precision top properties measurements provide an important test of the Standard Model (SM). The measurements of the top quark mass, charge and $\mathrm{W}$ polarization, performed with proton-proton $(p p)$ data at $\sqrt{S}=7 \mathrm{TeV}$ collected by the ATLAS detector until 2011 will be presented. The most up to date combination of ATLAS and CMS results will be also presented.

\section{Top quark mass}

The top quark mass has been measured studying $t \bar{t}$ events both in the lepton+jets and in the fully hadronic channels using $p p$ data at $\sqrt{\mathrm{s}}_{\mathrm{s}}=7 \mathrm{TeV}$ and with an integrated luminosity of $1.04 \mathrm{fb}^{-1}$ and $2.04 \mathrm{fb}^{-1}$, respectively.

For both channels $t \bar{t}$ events have been generated using the Next-to-Leading Order (NLO) Monte Carlo program MC@NLO [7] with the NLO parton density function set CTEQ6.6 [8]. Parton showering and underlying event are modeled using the Herwig [9] and Jimmy [10] simulator.

\subsection{Analysis in the $1+$ jets channel}

The event selection is tuned to select $t \bar{t}$ pairs events with two top quarks, one hadronically dacaying and one leptonically decaying. It is mainly characterized by the requirement of an isolated lepton ( $e$ or $\mu$ ), missing transverse energy $\left(E_{T}^{\text {miss }}\right)$, two bquark jets and two light jets at high $\mathrm{p}_{\mathrm{T}}$. Two different analysis techniques based on the template method, a 1-dimensional (1d) and a 2-dimensional (2d) analysis, have been adopted.

In the $1 \mathrm{~d}$-analysis a set of $1 \mathrm{~d}$ distributions of the $R_{32}=\frac{m_{t o p}^{\text {had }}}{m_{W}^{\text {had }}}$ variable has been produced varying the value of the top quark mass. The variable $R_{32}$ has been chosen in order to decrease the contribution of jet energy scale (JES) systematic uncertainty.

In the $2 \mathrm{~d}$-analysis a set of $2 \mathrm{~d}$ template distribution has been produced varying at the same time the top quark mass and a global Jet Scale Factor (JSF). This last global JSF has been obtained averaging over all possible $\eta$ and $\mathrm{p}_{\mathrm{T}}$ values to best fit the data once applied to all jets. In this method, the JES systematic uncertainty on the top mass is reduced and partly incorporated into an additional statistical uncertainty regarding the 2-dimensional fit technique.

The two analysis strategies have been combined but the result does not improve the precision of the top quark mass measured from the $2 \mathrm{~d}$-analysis:

$$
m_{\text {top }}=174.5 \pm 0.6_{\text {stat }} \pm 2.3_{\text {syst }} \mathrm{GeV} \quad(e+\mu \text { channels combination })
$$

The main sources of systematic uncertainties affecting this measurements are related to the JES, the b-jet tagging, the initial and final state radiation (I/FSR) and the hadronization. [1] 


\subsection{Analysis in the full hadronic channel}

Only events with five jets with $\mathrm{p}_{\mathrm{T}}>55 \mathrm{GeV}$ and a sixth jet with $\mathrm{p}_{\mathrm{T}}>30 \mathrm{GeV}$ have been kept for the analysis. A further request of $2 \mathrm{~b}$-tagged jets has been applied. The Template Method has been also used for this analysis. The templates have been derived using MC generators for $t \bar{t}$ events and using the Event Mixing Algorithm data-driven technique for multijet background. This algorithm selects data events with 5 jets and adds to them another jet, the $6^{\text {th }}$ in order of $\mathrm{p}_{\mathrm{T}}$ coming from data sample selected with the requirement of at least 6 jets. This merged sample represents the multi-jets background sample. A binned likelihood fit has been used the find the final top quark mass result:

$$
m_{\text {top }}=174.9 \pm 2.1_{\text {stat }} \pm 3.8_{\text {syst }} \mathrm{GeV}
$$

The main systematic uncertainties affecting this analysis are JES, b-tag, I/FSR and background modeling systematics. [2]

\subsection{Combined results}

A combination of the ATLAS and CMS top quark mass measurements using data collected from proton-proton collisions at the center-of-mass energy $V_{\mathrm{s}}=7 \mathrm{TeV}$ has been performed using the Best Linear Unbiased Estimator (BLUE) method[11]. The analysis included in the combination have been performed on data samples of different integrated luminosity, up to $4.9 \mathrm{fb}^{-1}$. The resulting combination takes into account statistical and systematic uncertainties and their correlations. The result is shown in Figure 1. The total uncertainty is dominated by the systematic uncertainty and its main contributions are due to jet calibration, signal modeling and underlying event tuning. [3]

\section{Top Quark Charge}

For this analysis $t \bar{t}$ events in the lepton+jets channel have been selected using $p p$ data collected at $V_{s}=7 \mathrm{TeV}$ and corresponding to an integrated luminosity of $0.7 \mathrm{fb}^{-1}$. The selection criteria were similar to the ones used for the top quark mass analysis, except for the b-jet tagging requirement that was not applied to the initial selection. Two different analysis techniques have been adopted differentiated by the method used to evaluate the b-jet charge.

In Track Charge Weighting analysis the request of $2 \mathrm{~b}$-tagged jets has been added in the event selection. The b-jet charge has been defined as:

$$
Q_{b j e t}=\frac{\sum_{i} q_{i}\left|\vec{j} \cdot \overrightarrow{p_{l}}\right|^{1 / 2}}{\sum_{i}\left|\vec{j} \cdot \overrightarrow{p_{l}}\right|^{1 / 2}}
$$

where $i$ runs over the 10 highest pt charged tracks inside a cone of radius $\mathrm{R}=0.25$ around the $\mathrm{b}$ jet axis; this selection allows to strongly reduce the pileup impact. 


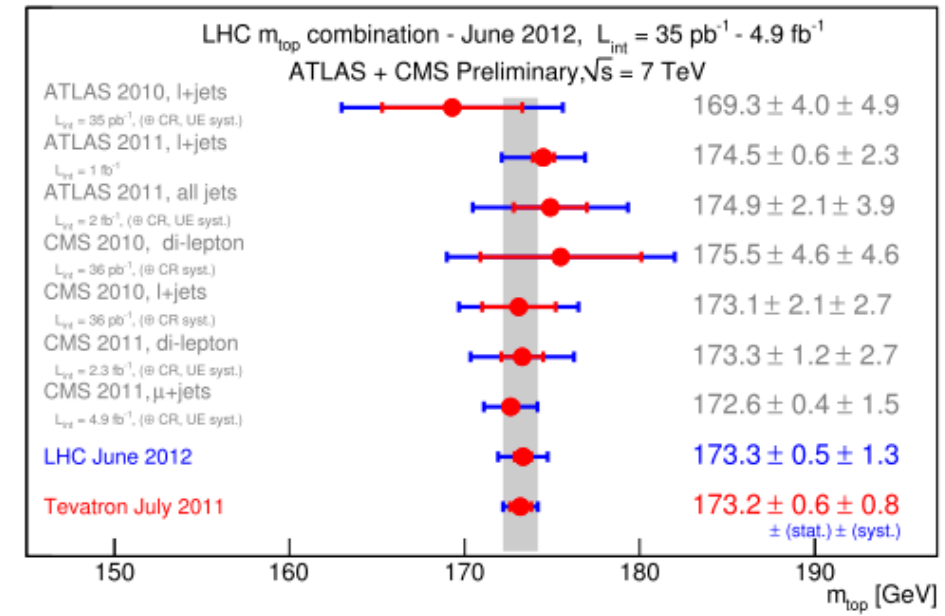

Figure 1- Combination of ATLAS and CMS top quark mass results. The combined Tevatron resultis also shown.
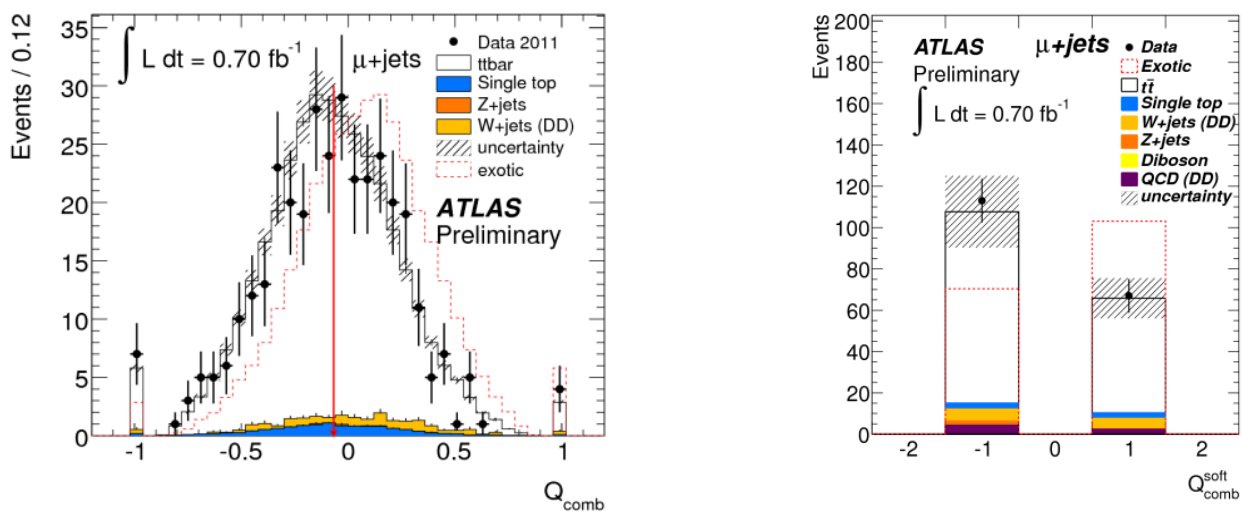

Figure 2 - $Q_{\text {comb }}$ distributions for the Track Charge Weighting (left) and the soft lepton method (right) compared with MC predictions and with the exotic top model( red dotted line).

In the Soft Lepton Method analysis at least one $\mathrm{b}$-jet with a muon inside a cone of $\mathrm{R}=0.4$ with respect to the jet axis has been required in order to select semileptonic decaying B-hadrons. The charge of this muon is the same of the original b-quark.

For both method the variable $\mathrm{Q}_{\text {comb }}$ has been compared with the one expected from $\mathrm{SM}$; it has been defined as $Q_{\text {comb }}=Q_{b j e t} \cdot Q_{\text {lepton }}$ where $Q_{\text {lepton }}$ is the charge of the lepton associated to the particular top decay process.

The most important contribution to systematics uncertainties came from JES and Monte Carlo modeling systematics. The final result is shown in Figure 2 and it is in good agreement with the Standard Model prediction in both cases. The exotic hypothesis of a "top-like" quark with charge $-4 \mathrm{e} / 3$ has been excluded at more than $5 \sigma .[4]$ 


\section{W boson polarization in $t \bar{t}$ decays}

This analysis has been performed on $p p$ data collected at $\sqrt{\mathrm{s}}_{\mathrm{s}}=7 \mathrm{TeV}$ and corresponding to an integrated luminosity of $1.04 \mathrm{fb}-1$. The lepton+jets selection is similar to the one described above (at least one b-jet) while the dileptonic selection requires two oppositely charged and isolated leptons, two jets, at least one of them should be b-tagged, plus some other kinematic and reconstruction cuts [6]. For the polarized signal generation the leading order (LO) Monte Carlo generator Protos [12] has been used with the CTEQ6L1 [13] sets of parton distribution functions (PDFs).

The polarization state of a real $\mathrm{W}$ boson can be longitudinal, right-handed or left-handed and depends on the Wtb electroweak vertex structure. The fractions of events with a particular polarization, $\mathrm{F}_{0}$ (longitudinal), $\mathrm{F}_{\mathrm{L}}$ (left-handed) and $\mathrm{F}_{\mathrm{R}}$ (right-handed), are referred to as helicity fractions and may be extracted from the event angular distribution parameterized as follows:

$$
\frac{1}{\sigma} \frac{d \sigma}{\cos \theta^{*}}=\frac{3}{4}\left(1-\cos ^{2} \theta^{*}\right) F_{0}+\frac{3}{8}\left(1-\cos \theta^{*}\right)^{2} F_{L}+\frac{3}{8}\left(1+\cos \theta^{*}\right)^{2} F_{R}
$$

The $\vartheta^{*}$ angle is defined as the angle between the momentum direction of the charged lepton from the decay of the $\mathrm{W}$ boson and the reversed momentum direction of the b-quark from the decay of the top quark, both boosted into the $\mathrm{W}$ boson rest frame. The polarization state could be also found measuring the angular asymmetry parameters A+ and A- defined as follows $\left(z= \pm\left(1-2^{2 / 3}\right)\right)$

$$
A_{ \pm}=\frac{N\left(\cos \theta^{*}>z\right)-N\left(\cos \theta^{*}<z\right)}{N\left(\cos \theta^{*}>z\right)+N\left(\cos \theta^{*}<z\right)}
$$

Both these ways have been followed and both the measurements have been performed using the Template Method. Several sources of systematic uncertainty has been considered and the most relevant are JES, lepton mismatch and the one depending on the particular method used in the analysis. The results [5] appear in good agreement with the theoretical prediction and with previous results. A combination, performed using the BLUE method, of ATLAS and CMS results has been also presented [6].

$$
\begin{gathered}
F_{0}=0.67 \pm 0.03_{\text {stat }} \pm 0.06_{\text {syst }}[\text { theory: } 0.687 \pm 0.005] \\
F_{L}=0.32 \pm 0.02_{\text {stat }} \pm 0.03_{\text {syst }}[\text { theory: } 0.311 \pm 0.005] \\
F_{R}=0.01 \pm 0.01_{\text {stat }} \pm 0.04_{\text {syst }} \quad[\text { theory: } 0.0017 \pm 0.0001]
\end{gathered}
$$

\section{Conclusion}

The measurements of top quark properties are very important in order to test the Standard Model. The ATLAS and CMS combined top quark mass result is in agreement with the previous Tevatron result. The top charge has been measured and does not show deviation 
from the expectation; an exotic "top-like" quark with charge $-4 \mathrm{e} / 3$ has been excluded at more than $5 \sigma$. The measurement of the $\mathrm{W}$ boson polarization in $t \bar{t}$ events is in agreement with the theoretical prediction and a limit on the Wtb vertex anomalous coupling has been placed.

\section{References}

[1] ATLAS Collaboration, Measurement of the Top Quark Mass with the Template Method in the $t \bar{t} \rightarrow$ lepton+jets Channel using ATLAS Data, Eur.Phys.J. C72 (2012) 2046.

[2] ATLAS Collaboration, Determination of the Top Quark Mass with a Template Method in the All-Hadronic Decay Channel using 2.04 fb ${ }^{-1}$ of ATLAS Data, ATLAS-CONF-2012-030.

[3] ATLAS and CMS Collaboration, Combination of ATLAS and CMS results on the mass of the top quarkusing up to $4.9 \mathrm{fb}^{-1}$ of data, ATLAS-CONF-2012-095.

[4] ATLAS Collaboration, Measurement of the top quark charge in pp collisions at $\sqrt{ } \mathrm{s}=7 \mathrm{TeV}$ in the ATLAS experiment, ATLAS-CONF-2011-141.

[5] ATLAS Collaboration, Measurement of the W boson polarization in top quark decays with the ATLAS detector, JHEP 1206 (2012) 088.

[6] ATLAS and CMS Collaboration, Combination of the ATLAS and CMS measurements of the Wboson polarization in top-quark decays, ATLAS-CONF-2013-033.

[7] S. Frixione and B.R. Webber, Matching NLO QCD computations and parton shower simulations, J. High Energy Phys. 06 (2002) 029

[8] M. Nadolsky et al., Implications of CTEQ global analysis for collider observables, Phys. Rev. D78 (2008) 013004.

[9] G. Corcella et al., HERWIG 6: An Event generator for hadron emission reactions with interfering gluons (including supersymmetric processes), J. High Energy Phys. 01 (2001) 010.

[10] J.M Butterworth, J.R. Forshaw and M.H. Seymour, Multiparton interactions in photoproduction at HERA, Z. Phys. C72 (2006) 637.

[11] A. Valassi, Nucl. Instrum. Meth. A500 (2003)391, doi:10.1016/S0168-9002(03)00329-2.

[12] J. A. Aguilar-Saavedra et al., Probing anomalous Wtb couplings in top pair decays, Eur. Phys. J. C 50 (2007) 519, [hep-ph/0605190].

[13] J. Pumplin et al., New generation of parton distributions with uncertainties from global QCD analysis, JHEP 07 (2002) 012, [hep-ph/0201195]. 